Archéologie du passé, mélancolie du présent - II

\title{
Spectrographie filmique de l'utopie : pour une mélancolie active des images en mouvement
}

\section{Raphaël Szöllosy}

\section{OpenEdition}

\section{Journals}

Édition électronique

URL : http://journals.openedition.org/grm/1812

ISSN : 1775-3902

Éditeur

Groupe de Recherches Matérialistes

Référence électronique

Raphaël Szöllosy, «Spectrographie filmique de l'utopie : pour une mélancolie active des images en mouvement ", Cahiers du GRM [En ligne], 15 | 2019, mis en ligne le 17 novembre 2019, consulté le 20 novembre 2019. URL : http://journals.openedition.org/grm/1812

Ce document a été généré automatiquement le 20 novembre 2019.

(c) GRM - Association 


\title{
Spectrographie filmique de l'utopie : pour une mélancolie active des images en mouvement
}

\author{
Raphaël Szöllosy
}

Même l'espoir déçu, qui erre et traîne son tourment comme un spectre égaré, loin du chemin du cimetière, est encore à la poursuite de ses rêves anéantis. (Ernst Bloch)․․

\section{Propos liminaire : d'une puissance mélancolique}

1 Lorsque Daniel Bensaïd publia son Pari mélancolique², il était déjà tard sur le cadran de l'horloge révolutionnaire. Si la forme que l'on avait connue de l'institution communiste s'était achevée quelques six années auparavant, il serait aisé de remonter bien plus tôt dans le temps pour goûter aux premiers sucs de la défaite et/ou de la trahison des espérances. Le verbe enragé d'Evguénia Iaroslavskaïa-Markon, rédigé de sa propre main dans un quartier disciplinaire et daté du 3 février 1931, permet ainsi de saisir un amer désenchantement, toutefois hautement combatif ${ }^{3}$, dès la première décennie qui suivît la révolution d'octobre. De ce fait, le siècle observé par Bensaïd, au sein duquel se sont levées tant de promesses, est jonché de multiples peines. Parmi les noms cités, de Walter Benjamin à Charles Péguy, de nombreux font partie de ces vaincus magnifiques qui auront porté les désirs d'un monde métamorphosé. "Des vaincus non point d'une défaite finale et définitive, mais pis encore, d'une défaite 'combien de fois recommencée', avec l'impression que rien n'est encore joué, que tout peut encore être rejoué $\rrbracket^{4}$ précise Bensaïd en se référant aux mots de l'auteur de Victor-Marie, Comte Hugo.

2 L'ambivalence au cœur d'un tel propos résume la fragilité d'un présent en attente d'émancipation qui connaît la conclusion parfois désastreuse des tentatives passées : dans la réussite d'une manœuvre de transformation sociale se tient la potentialité d'un 
échec à venir. Il semble à première vue aisé de sombrer dans cet état de dépréciation morbide qui aura été cliniquement associé à l'idée de mélancolie ${ }^{5}$. Pris par celui-ci, l'être fait l'expérience de la perte de son objet et de la perte du «moi » : son affliction est double et produit une dévalorisation de son existence qui bloque son rapport au monde. Il devient difficile alors d'imaginer poursuivre une bataille lorsqu'un idéal s'efface. Mais ce seuil de la défaite ne côtoie-t-il pas tous les présents du monde ? Les modèles de gouvernances qui maintiennent notre actualité dans une nuit terrifiante pourraient eux aussi chanceler; combien de Salazar et de colonels parviendront encore à prendre le pouvoir et combien chuteront devant nos yeux alors apaisés. La proposition peut alors se lire à l'inverse : derrière l'apparent succès des politiques honnies se tient la potentialité de l'émergence d'une alternative sociale. La lutte apparaît alors sans trêve $e^{6}$ si bien que la mélancolie ne peut pas ne pas nous guetter.

Mais, devrions-nous croire en une définition de la mélancolie qui la contraindrait dans un cadre thérapeutique - à une anesthésie de notre rapport à l'autre, à l'impossibilité de l'externalisation de soi, à l'abandon du souhait d'agir sur les choses environnantes? Bensaïd entrouvre une autre voie, celle d'une mélancolie active: "Mélancolie active toujours, irréductible au bord du désespoir, qui ne désarme pas " Cette " résistance sans illusion ${ }^{8}$ » aura touché plusieurs de ces figures référentielles qui nourrissent les écrits de l'auteur: "Mélancolie suicidaire de Benjamin, bien sûr, quittant Moscou en pleurs, sa valise sur les genoux, en 1927 ; tentant une première fois de se supprimer en 1933; y parvenant en 1940, les yeux écarquillés sur le désastre, terrassé par la nausée et la fatigue du siècle, devant le passage des Pyrénées où l'on ne passe plus »'. Celle aussi de Tucholsky, déclarant sans détour qu' « il faut tout reprendre à zéro. Il faut recommencer à zéro et ne pas écouter ce Staline ridicule qui trahit les siens comme seule le Pape sait le faire» avant de se questionner - "Est-ce que je décourage ?» - et de répondre "C'est déjà beaucoup de pouvoir éliminer les fausses et trompeuses espérances $»^{10}$. Mélancolies encore de Trotsky et Péguy qui «ont en commun un "mauvais caractère" qui ne plie pas devant l'échec, qui ne se résigne pas, qui se méfie des conforts et de la "réussite", des embourgeoisements et des bureaucratisations, qui refuse obstinément de s'incliner devant les connivences et les complicités compromettantes, de céder un peu aux raisons d'Etat avant de capituler beaucoup $»^{11}$.

Nul doute que l'image de la «bile noire » paraît adéquate pour décrire l'état d'âme de ces êtres ; ce ténébreux liquide aura même pu pousser certains d'entre eux à se donner la mort. Mais l'on peut incontestablement considérer cette sève mélancolique comme l'encre de leurs mots, dont la puissance les rend actifs jusque dans nos temps les plus actuels. Si nous considérons que le monde se porte mal, si nous n'éprouvons aucune joie quant à son état, alors les mélancoliques, habités d'un rapport critique à l'agencement des choses, ne peuvent être que des interlocuteurs privilégiés. En effet, dans le cas où l'on se tiendrait en opposition à l'organisation sociale telle qu'elle est, comment faire confiance aux euphoriques qui ne sont traversés d'aucun doute sur la méthode à employer pour maîtriser l'ordre des choses? Une hypothèse serait à penser : ne faudrait-il pas que les êtres au pouvoir s'arment de mélancolie pour ne pas chuter dans l'ivresse de leur exercice? Cette mélancolie active pourrait être un moyen de nous faire contemporains, dans le sens que donne Giorgio Agamben à ce terme :

La contemporanéité est donc une singulière relation avec son propre temps, auquel on adhère tout en prenant ses distances; elle est très précisément la relation au temps qui adhère à lui par le déphasage et l'anachronisme. Ceux qui coïncident trop 
pleinement avec l'époque, qui conviennent parfaitement sur tous les points, ne sont pas des contemporains parce que, pour ces raisons mêmes, ils n'arrivent pas à la voir. Ils ne peuvent pas fixer le regard qu'ils portent sur elle ${ }^{12}$.

5 La mélancolie ne peut-elle pas être cet écart au monde qui placerait le sujet qu'elle touche dans ce décalage propice à un regard attentif et lucide ? Rendre cet état " actif » pourrait passer par sa cristallisation à travers des formes, des mots ou des images. Ainsi les lecteurs et spectateurs seraient aptes à travailler leur propre contemporanéité en se confrontant aux objets issus d'autres temporalités. Songeons aux Cahiers de Prisons d'Antonio Gramsci comme à l'Eternité par les astres d'Auguste Blanqui rédigés dans l'une ou l'autre de ces nuits infinies: peut-on imaginer plus haut lieu de désespoir que le cachot? Ces œuvres ne nourrissent-elles pas toutefois nombre de contemporains qui s'enrichissent de leurs puissances théoriques et aiguisent leur distance quant aux velléités dominantes? Cette faculté de saisir ces signes animés par l'espérance d'une métamorphose du monde, malgré les désastres et par-delà le deuil, s'approche de l'idée « d'images-posthumes » chez Ernst Bloch.

\section{Des Images-posthumes contre la condition larvaire}

6 «À quoi nous serviront alors nos utopies et nos cultures, si tout doit finir dans le nihil, le néant, le nihilisme? ? questionne le philosophe de Tübingen, avant d'affirmer que " pour contrer cela, il existe encore des utopies et des tentatives pour indiquer des chemins qu'on rencontre sur la ligne de front qu'occupent les hommes, tout comme on trouve aussi de la duperie, des contes de fées et des rêves au mauvais sens du terme ${ }^{13}$. S'il faut se mettre en garde des illusions béates et des prêches fallacieuses, nous ne saurions admettre l'extinction définitive des imaginaires alternatifs malgré tous les désespoirs. La mort elle-même - d'un être, d'un sentiment, d'un idéal - ne peut être synonyme d'un ultime abandon: "L'image-posthume bien comprise, qui n'a plus rien de commun ni avec le culte des morts, ni avec une régression par jouissance rétrospective, peut être des plus fécondes, car elle rayonne dans ces sphères où le passé offre la promesse d'un non-devenu venant à notre rencontre $»^{14}$.

7 Certes, les dangers paraissent chaque jour trop grands: «Ce qui bloque, ce qui fait obstacle, la puissance persistante du négatif s'exprime de mille et une manières, aussi horribles les unes que les autres " affirme Bloch, bien lucide sur les multiples sources de nos mélancolies : «Le poison des maladies, l'exploitation et la répression avec leurs masques et leurs idéologies sans cesse renouvelés, les coiffant toutes " autant que «l'anonymat du capital, le fait que pour mille guerres il n'y ait guère que dix révolutions, qu'on puisse toujours brûler aussi facilement le Reichstag, que des révolutions réussies et marquant un nouveau commencement dégénèrent si inopinément en d'anciennes horreurs $\aleph^{15}$. Mais l'attraction vers la force du souhait qui anime chaque être lorsqu'il est insatisfait de sa condition ne peut pousser qu'à outrepasser la finitude : «La curiosité transforme le rideau qui tombe en rideau qui se déchire; et la fin de la vie apparaît alors comme le début de quelque-chose d'absolument inouï; même s'il s'agit du néant. La curiosité peut aussi passez à un niveau supérieur; celui du désir d'explorer et de connaître, elle attend alors du processus de la mort qu'il soit une révélation $»^{16}$. En suivant cette réflexion, la mort n'est pas réductible au nihil. Après elle se perpétue l'activité créatrice d'un être: 
«Quoiqu'il en soit et contrairement à la croyance en la dissolution dans le tout, la part la plus noble de l'esprit individuel peut être sauvé de la ruine générale $»^{17}$.

8 Le trépas, qui peut annihiler l'espoir porté par une révolution ou faire perdre l'être pour qui l'on éprouve le plus grand amour, est une source évidente de profonde mélancolie. Mais si le mélancolique estime que la mort n'est pas réductible au néant, ne peut-il pas s'employer à retrouver cette puissance active décrite par Bensaïd ? Ne peutil pas continuer à se confronter à cette "part noble de l'esprit " malgré tous les désarrois? Seulement, pour ce faire, il est nécessaire de fonder et de fréquenter des espaces où ce type de rencontres sont imaginables: «Des hommes meurent, des villes s'écroulent, des royaumes s'effondrent, mais la bibliothèque en recueille et en rassemble toutes les significations et les arrache par conséquent au domaine de l'éphémère $»^{18}$.

9 Nous postulerons ici que le cinéma peut être un de ces lieux où se consolide un héritage et où les images-posthumes peuvent se rendre visibles. Celles-ci outrepassent la finitude de la tombe sans même recourir à l'idéal d'un au-delà transcendant. La philosophie matérialiste de Bloch appréhende en effet les écrits religieux plutôt comme des fables illustrant les «images-souhaits» d'un horizon meilleur. Mais c'est bien au cœur des productions de l'homme que nous nous devons de saisir les surgissements d'après la mort, armés du désir de réinventer le monde. Le regard du penseur, imprégné de son "optimisme militant », pousse à s'extraire de la considération d'une "humanité enveloppée de sa nuit nihiliste», qui ne serait alors "plus qu'une apparition phosphorescente, ou alors, enveloppée de vide, fluorescente un tube de Geissler $»^{19}$. Pour ce faire nous sommes amenés à nous libérer de toutes craintes, car seul «l'homme qui a peur des fantômes se sent la proie d'un danger ${ }^{20}$ ». En effet, les « images-posthumes » seraient l'occasion d'un entretien avec les spectres.

Giorgio Agamben réfléchit précisément à cette figure dans un court texte qui démarre sur un constat livré à propos de la ville de Venise. La capitale de la Vénétie «n'est plus qu'un cadavre $\|^{21}$ affirme d'abord le philosophe italien. Mais puisqu'elle continue malgré tout à exister d'une manière ou d'une autre, ce ne peut être que « dans l'état qui suit la mort et la décomposition du cadavre. Cet état, c'est le spectre $»^{22}$. Un tel état est notamment visible «le long des Zattere désertes [où] la Giudecca, comme si elle bredouillait, rejette sur les parvis des algues moisies et des bouteilles en plastique ${ }^{23}$. La première exigence que suppose le caractère post-cadavérique de Venise consiste en une reconnaissance et en un effort affectif : « Aimer une morte est chose difficile. Il est plus facile de feindre qu'elle est encore en vie, de couvrir ses membres délicats et exsangues avec des déguisements et des maquillages pour pouvoir les montrer aux touristes contre de l'argent $»^{24}$.

11 Cependant, nous ne saurions réduire l'idée de "spectre » à l'absolu du néant. Au contraire, « la spectralité est une forme de vie. Une vie posthume ou complémentaire, qui commence seulement une fois que tout est fini et qui a donc, par rapport à la vie, la grâce et l'astuce incomparable de ce qui est achevé, l'élégance et la précision de ce qui n'a plus rien devant soi $»^{25}$. Cette vision positive du fantôme est liée à une certaine exigence et elle fait face à un dangereux pendant: «Il y a cependant une spectralité d'un autre type, que nous pouvons appeler larvaire ou larvée, qui naît chez ceux qui refusent leur condition et tentent de les refouler pour feindre à tout prix qu'ils sont dotés d'un poids et d'une chair $\aleph^{26}$. La spectralité prendrait alors la forme d'un embryon continu et deviendrait une hantise négative, capable de se mouvoir de façon fallacieuse 
au sein des êtres. Venise est pour Giorgio Agamben l'exemple d'un tel caractère larvaire qui refoule sa mort sous les artifices publicitaires. Mais si l'état de la ville inquiète le philosophe, c'est parce qu'elle est à l'image de son temps : «Notre époque n'est pas nouvelle, mais novissima, c'est-à-dire dernière et larvaire » déclare-t-il ainsi, « elle s'est conçue comme posthistorique et comme postmoderne, sans penser qu'elle se destinait ainsi nécessairement à une existence posthume et spectrale $»^{27}$. Tout cela révèle justement certains aspects de notre situation politique dont profitent sans doute les plus récentes formes de fascisme dans de nombreux endroits du monde : c'est pourquoi « les parlementaires légifèrent en vain, parce qu'ils doivent faire croire en une vie politique à leur nation larvaire", c'est pour cette raison que "des momies qui prétendent diriger allégrement leur exhumation sans s'apercevoir que leurs membres décomposés les abandonnent pour tomber en morceaux et que leurs paroles sont devenues des glossolalies inintelligibles $\aleph^{28}$. Cette condition larvaire ressemble alors à la fois à la mélancolie dépréciative et à sa faculté d'incube ou de succube ainsi qu'à un certain esprit du temps, fait de déni, de spectacle et de cadavres en décomposition.

Malgré tout, la conclusion du texte de Giorgio Agamben esquisse une forme de principeespérance:

De tout cela le spectre de Venise ne sait rien. Ce n'est plus aux Vénitiens, et certes pas davantage aux touristes qu'il pourrait apparaître. C'est peut-être aux mendiants et aux administrateurs sans vergogne qui veulent les chasser, peut-être aux rats qui traversent les calles à toute vitesse, museau contre terre, ou peut-être à ces rares personnes, exilées, presque, qui essaient d'en élaborer la fuyante leçon. Parce que ce que le spectre soutient de sa voix blanche, c'est que, à partir du moment où toutes les villes et toutes les langues d'Europe survivent désormais comme des fantômes, il n'appartiendra qu'à ceux qui auront su inventer avec eux un rapport intime et familier, épeler à nouveau et mémoriser leurs paroles décharnées et leurs pierres, de voir à nouveau s'ouvrir ce passage où l'histoire - la vie - remplit brusquement ses promesses ${ }^{29}$.

13 C'est à ceux qui habitent les marges du monde et à ceux qui accepteraient de construire un rapport privilégié avec les esprits des temps passés que peut apparaître la puissance positive des spectres. Une mélancolie active peut à la fois forger notre écart au terrible déroulement des choses et nous pousser à l'écoute fantomatique de ceux qui sont venus avant nous et auront cristallisé leurs pensées résistantes. Car telle est la nécessité de l'établissement d'une mémoire capable d'ébrécher les logiques dominantes: «Toute image posthume est sujette à caution, si elle n'a pas pu se constituer à l'époque même où son objet vivait encore, car ce n'est qu'alors que son halo peut rayonner d'un éclat authentique $\aleph^{30}$. Une autre attention est à porter dans notre relation à cet imaginaire des temps antérieurs. Il y aurait à prendre garde de ne pas porter des costumes tissés de cette peau larvaire et de ne pas se complaire dans une nostalgie délétère en lieu et place de cette mélancolie active dont on esquisse ici la potentialité: "L'imageposthume célébrée à tort exclut toute vie nouvelle au profit d'une vie passée qui se prolonge dans un présent inauthentique, entrainant l'inconvénient de ce que l'on peut appeler, dans l'optique de l'âme également, reflet réitéré »" écrit ainsi Ernst Bloch. Mais si le rapport au spectre exclut tout fantasme morbide et témoigne d'un désir de renouveau alors « un nouvel espoir nait du souvenir et l'image posthume offre une promesse $»^{32}$. De cette façon « la bien-aimée défunte s'est libérée de l'emprise du simple souvenir, la nostalgie éveillée par l'imago n'est plus stérile, mais luit comme une étoile au firmament de l'avenir $»^{33}$. 

faire rencontrer ces spectres d'une façon positive et émancipatrice. Le cinéma est l'un de ces vecteurs d'esprits capables de les rendre "actifs", ne serait-ce qu'imaginairement, dans les temps contemporains. C'est à travers l'étude d'une œuvre en particulier et des fantômes qui lui sont attachés que nous tenterons de saisir cette idée.

\section{Spectrographie : autour de Ghost Dance (1983) de Ken McMullen}

Il est fort périlleux de tenter de résumer Ghost Dance de Ken McMullen qui fut pour la première fois diffusé sur une télévision ouest-allemande à la fin de l'année 1983. Nulle ligne narrative déterminée ne structure sa progression. Il s'agit plutôt d'un montage de séquences disparates et de réflexions, poétisées par la forme de l'œuvre, sur la technique, la politique, ou le cinéma lui-même, qui gravitent autour de deux femmes : Pascale jouée par Pascale Ogier rencontre Marianne interprétée par Leonie Mellinger, et l'une semble se refléter dans l'autre. Plusieurs paysages sont traversés, d'une usine désaffectée à une mer agitée, d'un toit d'un borough londonien à un monument à la mémoire de la Commune de Paris. Au cœur de ces entrelacs, la problématique de la figure du fantôme s'incarne dans la présence de Jacques Derrida au sein du film, interprétant son propre rôle. Pascale, étudiante en philosophie, est amenée à le rencontrer.

L'un des nœuds de Ghost Dance se situe dans une scène improvisée où les deux individus se font face dans le bureau de Derrida. "Je voudrais vous demander une chose, est-ce que vous croyez aux fantômes?». C'est de cette manière que l'actrice s'adresse au professeur qui propose son raisonnement: "Je ne sais pas, c'est une question difficile. D'abord est-ce qu'on demande à un fantôme s'il croit aux fantômes? » questionne-t-il d'abord, en revendiquant ainsi sa dimension spectrale dès lors qu'il se joue de lui-même dans un film: "J'ai l'impression de laisser parler un fantôme à ma place; paradoxalement, au lieu de jouer mon propre rôle, je laisse à mon insu un fantôme me ventriloquer, c'est-à-dire parler à ma place ». C'est bien l'appareil cinématographique qui se trouve problématisé : «Le cinéma, quand on ne s'y ennuie pas, c'est ça : c'est un art de laisser revenir les fantômes ». Renouer le contact avec les spectres parait indissociable d'une réflexion sur la technique. Jacques Derrida affirme de ce fait "qu'aujourd'hui, tout le développement de la technologie des télécommunications, au lieu de restreindre l'espace des fantômes» leur attribue la responsabilité de «l'avenir ». "Je crois que l'avenir est aux fantômes » déclare-t-il avec conviction, «la technologie moderne de l'image, de la cinématographie, de la télécommunication, décuple le pouvoir des fantômes, le retour des fantômes ». Telle est la raison de sa propre apparition dans Ghost Dance: "C'est au fond pour tenter les fantômes que j'ai accepté de figurer dans un film, en me disant que peut-être on aurait, les uns et les autres, la chance de laisser venir à nous des fantômes : fantôme de Marx, fantôme de Freud, fantôme de Kafka, fantôme de cet américain...Vous!» développe-t-il, amusé, face à Pascale Ogier, avant de lui retourner la question : «Je ne sais pas si je crois ou si je ne crois pas aux fantômes, mais je dis 'Vive les fantômes' ! Et vous, est-ce que vous y croyez aux fantômes?».

Cahiers du GRM, 15 | 2019 
17 Nous entendons sa réponse au moins trois fois - "Mais certainement », "Oui, absolument ", "Maintenant, absolument" - manière de révéler le caractère reproductible inhérent au cinéma. Mais au-delà du discours, c'est l'image même de Pascale Ogier au sein de Ghost Dance, qui amplifie le rapport aux spectres: l'actrice mourra en effet tragiquement le 25 octobre 1984 à la veille de ses 26 ans. Dans un entretien avec Bernard Stiegler, Jacques Derrida revient alors sur la singulière expérience de revoir la scène après un tel évènement :

Mais imaginez quelle a pu être mon expérience quand, deux ou trois ans après, alors que Pascale Ogier, dans l'intervalle, était morte, j'ai revu le film aux EtatsUnis, à la demande d'étudiants qui voulaient en parler avec moi. J'ai vu tout à coup arriver sur l'écran le visage de Pascale, que je savais être le visage d'une morte. Elle répondait à ma question: "Croyez-vous aux fantômes?». En me regardant quasiment dans les yeux, elle me disait encore, sur grand écran : «Oui, maintenant, oui ». Quel maintenant? Des années après au Texas. J'ai pu avoir le sentiment bouleversant du retour de son spectre, le spectre de son spectre revenant me dire, à moi ici maintenant: "Maintenant...maintenant, c'est-à-dire dans cette salle obscure d'un autre continent, dans un autre monde, là, maintenant, oui, crois-moi, je crois aux fantômes ${ }^{34}$.

Les images en mouvement ont la capacité de perturber le cours du monde. L'enregistrement du passé par les moyens du film complexifie le rapport spatial et temporel du spectateur. De cette façon Jacques Derrida expérimente la possibilité d'une conversation renouvelée avec un fantôme. Mais selon le philosophe cet état spectral était déjà à l'œuvre au moment où Pascale Ogier s'exprimait devant la caméra :

Mais en même temps je sais que la première fois où Pascale a dit cela, où elle a répété cela dans mon bureau, déjà, déjà cette spectralité était à l'œuvre. Elle était déjà, elle disait déjà cela, et elle savait, comme nous savons, que même si elle n'était pas morte dans l'intervalle, un jour, c'est une morte qui dirait : «Je suis mort » ou : "Je suis morte, je sais de quoi je parle d'où je suis, et je te regarde », et ce regard restait dissymétrique, échangé au-delà de tout échange possible, eye-line sans eyeline, eye-line d'un regard qui fixe et cherche l'autre, son autre, son vis-à-vis, l'autre regard croisé, dans une nuit infinie ${ }^{35}$.

19 Toute forme vivante est destinée à connaitre le trépas. Le fait même de se transformer un instant en image change en spectre celui dont les mouvements seront reproduits. De nombreuses occasions sont données de traverser des deuils et multiples sont les abattements liés à la mélancolie. Cependant à la manière d'Ernst Bloch, il y aurait à apprendre à ne pas craindre le devenir spectral de l'être. Envisager avec confiance et sérénité cette potentialité contraindrait peut-être la condition larvaire et la dépréciation morbide. Mais encore faut-il que le spectre puisse avoir un lieu pour se réfugier : ainsi pourrions-nous considérer la capacité spectrographique du cinéma ${ }^{36} \mathrm{en}$ regard du contexte de la mélancolie politique la plus contemporaine. Car en tant que «mémoire spectrale», «le cinéma est un deuil magnifique», un travail de deuil magnifié. Et il est prêt à « se laisser impressionner par toutes les mémoires endeuillées, c'est-à-dire par les moments tragiques et épiques de l'histoire $»^{37}$. Il a donc fort à faire concernant l'époque que nous traversons, éprouvée par le travail du deuil d'espérances déçues et la construction de nouvelles formes d'avenirs. Derrida lui-même aura pu poser la problématique de la spectralité sur le temps qui allait s'ouvrir après la chute de l'Union soviétique.

Dix années après Ghost Dance, le philosophe publie Spectres de Marx (1993). Et dans ce monde «désajusté », fait d'un «maintenant disjoint», qui désormais «sort de ses 
gonds $\aleph^{38}$, il ne lui semble pas possible de compter sans la présence des fantômes, au nom d'une certaine « justice $»^{39}$ :

Il faudrait alors apprendre les esprits. Même et surtout si cela, le spectral, n'est pas. Même et surtout si cela, ni substance ni essence ni existence, n'est jamais présent comme tel. Le temps de l'“apprendre à vivre", un temps sans présent tuteur, reviendrait à ceci, l'exorde nous y entraîne : apprendre à vivre avec les fantômes, dans l'entretien, la compagnie ou le compagnonnage, dans le commerce sans commerce des fantômes. À vivre autrement, et mieux. Non pas mieux, plus justement. Mais avec eux. Pas d'être-avec l'autre, pas de socius sans cet avec-là qui nous rend l'être-avec en général plus énigmatique que jamais. Et cet être-avec les spectres serait aussi, non seulement mais aussi une politique de la mémoire, de l'héritage et des générations ${ }^{40}$.

Un tel apprentissage, peut-être nécessaire à tout projet de construction sociale dans un présent qui ne répond plus au nom d'une figure référentielle émancipatrice, amène à une certaine forme de "politique de l'amitié » avec les esprits qui pousse celui qui fait l'expérience de cette compagnie à transmettre les pensées antérieures à lui dans l'optique d'un au-delà du contemporain. Le " "savant" de l'avenir », «l'“intellectuel" de demain", et sans doute le mélancolique actif se doivent de "s'entretenir » avec le fantôme, ils ont « à lui laisser où à lui rendre la parole, fût-ce en soi, en l'autre, à l'autre en soi : ils sont toujours là, les spectres, même s'ils n'existent pas, même s'ils ne sont plus, même s'ils ne sont pas encore ${ }^{41} »$.

\section{L'utopie de la commune des esprits.}

Il y a de fait nombre de spectres qui tiennent compagnie à Ghost Dance de Ken McMullen. Et ceux-ci sont fort susceptibles d'être attachés à l'idée de mélancolie. Lorsqu'au sein du film les deux personnages féminins se parent des attributs de guerrières issues des sociétés traditionnelles, l'image rejoint figurativement l'esprit que l'œuvre revendique jusque dans son titre même. La Ghost Dance est en effet une pratique culturelle et cultuelle amérindienne, plus précisément liée aux Lakotas Minconjous, qui se déploya face à l'emprise des colons et à la mort de ceux qui résidaient jusqu'alors sur les terres américaines. Le massacre de Wounded Knee est le moment historiquement marquant de cette danse aux attentes messianiques:

Juste après 9 h30 le 29 décembre 1890, la fusillade commença. L'après-midi précédente, les soldats de la cavalerie des Etats-Unis avaient intercepté les Lakotas Miniconjous de Bigfoot et les avaient forcés à camper le long de la Wounded Knee Creek dans l'Etat du Dakota du sud. Comme de nombreux autres Lakotas, le peuple de Bigfoot avait adopté une religion qui avait émergé au sein de la réserve de la Walker River, dans l'Ouest du Nevada, environ deux ans auparavant. C'est là que Wovoka, le prophète Paiute du Nord, enseigna aux fidèles que s'ils pratiquaient les rituels prescrits et conduisaient d'honnêtes et paisibles vies, ils seraient bientôt réunis avec leurs amis décédés et les êtres chers dans une terre ressuscitée. Les observateurs blancs alors contemporains nommèrent cela la religion de la danse des esprits (Ghost Dance), ou, plus communément, ils se referaient de façon dérisoire à cette foi simplement en tant qu' " engouement du messie » (Messiah craze). Parmi les Lakotas la danse était devenue une danse de résistance. De nombreux danseurs portaient des "chemises fantômes" (ghost shirts), qui selon leur croyance les protégeaient des balles ${ }^{42}$.

Malgré leur ferveur, les Lakotas Minconjous rejoignaient leurs êtres chers dans une fosse commune, sans réussir à refonder une communauté avec eux sur cette terre. De 
cette manière, la danse des esprits a pu devenir « une métaphore des attentes désespérés et illusoires d'un peuple à retrouver l'irretrouvable » et l'expression "des finitudes, du désespoir et de la mort $»^{43}$. Malgré cette sombre tragédie issue de la plus haute des cruautés, il serait bien trop réducteur de sacrifier la Ghost Dance sur l'autel de la dépréciation morbide. Gregory Ellis Smoak affirme ainsi que le rite des Lakotas "n'était pas une fantaisie religieuse désespérée qui aurait surgi et aurait été rapidement abandonnée après l'échec d'un retour de l'âge d'or prophétique, mais une ancienne cérémonie $~_{44}$. Il précise que « lors des premières années de vie en réserve, la Ghost Dance était devenue une partie d'une réponse religieuse de longue date à la colonisation, réponse ancrée dans des pratiques culturelles préexistantes et façonnée par l'émergence d'une identité ethnique et raciale $»^{45}$. Avant même la prophétie de Wovoka - aussi connu sous le nom de Jack Wilson - venue à lui lors de l'éclipse du jour de l'an $1889^{46}$, une telle pratique avait pu être décelée dès les années 1870 en tant que premier mouvement pan-amérindien émergeant $d u$ Grand Bassin ${ }^{47}$. Le prêcheur s'appelait alors Wodziwob et visait un "retour des chemins passés, l'abondance du gibier comme de la nourriture végétale et tous les indiens, vivants et morts, réunis sur une terre renouvelée $»^{48}$. Bien que "le message [fût] révolutionnaire», "les rituels étaient assez traditionnels. La cérémonie au socle de la Ghost dance de 1870 autant que celle de 1890 était l'ancestrale ronde Païute $~^{49}$ à laquelle s'ajoute l'inspiration de la chrétienté qui «a probablement transformé la croyance préalable en regard de la désirabilité de la fin du monde et du retour des morts » ${ }^{50}$.

La pratique de 1890 est donc elle-même une réinvitation de celle survenant vingt années auparavant, elle-même construite autour de la mémoire d'une culture traditionnelle et de la réappropriation de certains éléments de l'oppresseur. Spectralité multiple, donc. Et spectrographie à venir : la firme d'Edison par le biais du kinétographe aura filmé une danse des esprits quatre années après le massacre de Wounded Knee, un an avant le cinématographe des Frères Lumière dans Sioux Ghost Dance ${ }^{51}$ (1984). En bas à droite du cadre un écriteau permet de préciser le contexte du film, produit par W. K-L Dickson. Les indiens qui s'animent à l'écran font partie de la troupe du Buffalo Bill's Wild West Show et furent filmés par William Heise le 24 septembre 1894 dans le studio Black Maria d'Edison. Mélancolique constat que de voir ces êtres soumis à la société du spectacle aux lendemains du massacre de leurs proches ${ }^{52}$. Mais l'activité spectatorielle peut cependant refuser de circonscrire les êtres à cet état de fait et saisir l'occasion de cette spectrographie pour éveiller la mémoire de ces luttes, aussi endeuillées soientelles. Car le cri de cet enfant en bas à gauche du cadre peut bien rester muet, il n'est pas sans résonnance imaginaire.

Edison lui-même s'est intéressé à la possibilité d'un dialogue avec les fantômes. Il consacrera ainsi le dernier chapitre de ses Mémoires et observations, publiées à titre posthume en 1948, à une telle théorie sur laquelle reviennent Philippe Baudoin et Mireille Berton: "La "machine nécrophonique" de l'inventeur américain serait en mesure de détecter les paroles de cette personnalité transformée en ce qu'il appelle des "unités de vie" qui vibreraient et se disperseraient à travers l'éther " ${ }^{53}$. Si même pour l'ingénieux aux mille brevets l'affaire semble complexe à concrétiser, sa quête n'aura pas finalement pas été sans résultat: les deux auteurs proposent de considérer les appareils d'enregistrement inventés par Edison - le kinétoscope, le kinétophone ou le phonographe comme des "actualisations de ses "machines à fantômes" jamais réalisées ${ }^{54} »$. 

possibles où nous serions à même de nous entretenir avec les spectres, ainsi que Derrida nous invite à le faire. Le cinéma est alors traversé par l'idée d'une utopie en acte : le monde qui s'y offre est à la fois des plus évanescents - il n'apparait que pendant un temps donné et sa présence n'est qu'imaginaire, il s'approche ainsi du sens étymologique du non-lieu - mais l'existence de son principe - fondé autour de l'enregistrement, du montage et de la projection en un espace donné, intime ou collectif - est des plus concrètes. Outre la mémoire de ces danses amérindiennes, Ghost Dance est justement travaillé par l'utopie de la Commune de Paris qui connut une existence bien réelle.

Dans la troisième partie du film - intitulée " Histoire. Fantômes des rêves éveillés » surgit la mémoire de l'évènement. Au sein d'une pièce aux murs décrépis, Marianne reçoit Pascale. Autour d'elles de multiples affiches rappellent l'insurrection parisienne. Un souvenir mène les deux protagonistes devant le tombeau parisien commémorant les morts du 21 au 28 mai 1871 avant que le personnage joué par Dominique Pinon ne livre un discours en tant qu'occasionnel guide touristique. Il démarre alors par la tragédie de la semaine sanglante: «La Commune était la première révolution prolétarienne. Les Communards combattirent courageusement. Dans les rues de Paris, derrière les barricades, ils tombèrent jusqu'au dernier. Les femmes, les enfants et les hommes ». C'est cependant en s'opposant à l'annihilation de l'utopie qu'il conclut son discours :

En dépit de ces horreurs, ils n'ont pas réussi à tuer l'idée. L'idée continue à vivre, comme c'est souvent le cas. Personne ne peut tuer une idée. Les idées ont des ailes et volent dans l'espace éternel.

Loin de toute anesthésie du possible, Kristin Ross, dans son travail sur l'imaginaire de la Commune, expose le même type de réflexion. Pour l'auteure, l'évènement, ce «laboratoire d'inventions politiques, improvisées sur place ou bricolées à partir de scénarios ou d'expressions du passé, repensée selon les besoins du moment, et nourris des désirs nés au cours des réunions populaires de la fin de l'Empire $»^{55}$, provenait déjà d'un compagnonnage avec quelques spectres :

C'était une façon d'être intensément dans le présent, rendue possible par la mobilisation de figures et d'expressions du passé - à commencer par celle de commune elle-même, dont la charge affective déborde tout contenu sémantique précis, combinaison puissante de désirs précapitalistes et pré - ou extra-nationaux, à la fois révolution sociale, autonomie locale et mémoire de la Commune insurrectionnelle qui avait fait de Paris la capitale de la révolution en $1792^{56}$.

L'évènement de la Commune - aujourd'hui référence majeure dans bien des actes militants, porteurs de la ferveur de son existence comme de la mélancolie de son sanglant échec - n'a, selon Kristin Ross, rien d'un pur surgissement mais se conçoit plutôt comme traversé par de multiples fils mémoriels et spatiaux. Car « la Commune n'était pas tant un événement de l'histoire nationale française » suggère par ailleurs l'auteure à partir du travail de Benoît Malon, «qu'un des éléments d'un vaste tableau mondial qui comprenait la révolte indienne contre le capitalisme britannique, la libération des esclaves nord-américains, les révoltes en Irlande, en Hongrie, en Pologne et le développement de l'opinion libérale en Russie, présage d'une lueur de liberté pour les serfs $»^{57}$.

Un tel entrelacement paraît également se porter au regard du spectateur contemporain de Ghost Dance. Dans une séquence du film, alors que la caméra balaye le champ de droite à gauche avant de faire marche arrière, Leonie Mellinger et Pascale Ogier, 
d'abord affublée d'un masque traditionnel, nous font face. Derrière elles apparaissent la célèbre photographie des communards exécutés, attribuée à Adolphe-Eugène Disdéri, et une affiche du Comité du salut public. Sur ces images, se posent la voix de Jacques Derrida puis le drone musical de David Cunningham. L'ensemble de ces éléments - la mémoire amérindienne, celle des insurgés de Paris, les figures du penseur et des actrices - se mélange et forme ici une commune spectrographique, une commune des esprits capable de s'adresser de façon utopique aux spectateurs.

Dans cette séquence, le philosophe y précise la théorie du deuil de Nicolas Abraham et Maria Torok, qui résonne avec la distinction entre les spectres et les larves énoncée par Giorgio Agamben. Lors d'un travail de deuil « réussi » nous serions à même d'accueillir le mort, de l'intérioriser. Mais lorsque que ce deuil échoue, le mort s'incorpore à nous « et il peut parler tout seul, il peut hanter ou ventriloquer notre propre corps, notre propre discours ». Il nous semble que cette distinction touche l'idée de mélancolie et peut aider à saisir les enjeux politiques du contemporain. Le type de relation que l'on entretient avec le posthume pourrait ainsi déterminer si la mélancolie est faite de dépréciation morbide et d'abandon du souhait de construire un commun, ou au contraire si elle peut se révéler en tant que " germe de la lucidité dans la catastrophe et la modernité » et mue par une "capacité d'éveil " ${ }^{58}$. Dès lors, il s'agirait moins de remettre en scène tel ou tel événement révolutionnaire dans le temps présent que d'accueillir en nous leurs esprits pour imaginer de nouvelles méthodes, de nouvelles formes, de nouvelles voies habitées pas la mémoire et l'Histoire des actes passés.

«L'avenir est aux fantômes » nous dit Jacques Derrida dans Ghost Dance. Peut-être cet avenir appartient aussi aux "veilleurs et aux éveilleurs " ${ }^{59}$, nécessairement mélancoliques, de ce monde. En tant que spectrographe, le cinéma - qui cristallise en les reproduisant les espaces et les temps - est susceptible d'être une source d'utopies concrètes à laquelle ils pourraient s'abreuver.

\section{NOTES}

1. Ernst Bloch, Le Principe-Espérance, t. 1, traduit de l'allemand par Françoise Wuilmart, Paris, Gallimard, 1976, p. 237.

2. Daniel Bensaïd, Le pari mélancolique, Paris, Fayard, 1997.

3. Evguénia Iaroslavskaïa-Markon, Révoltée, traduit du russe par Valéry Kislov, Paris, Seuil, 2017.

4. Daniel Bensaïd, op. cit., p. 254.

5. Sigmund Freud écrit ainsi : "Psychiquement, la mélancolie se caractérise par une humeur profondément douloureuse, un désintérêt pour le monde extérieur, la perte de la faculté d'amour, l'inhibition de toute activité et une autodépréciation qui s'exprime par des reproches et des injures envers soi-même et qui va jusqu'à l'attente délirante du châtiment. Nous comprenons mieux ce tableau quand nous considérons que le deuil présente les mêmes caractéristiques, à l'exception d'une seule: l'autodépréciation morbide.» dans Deuil et mélancolie, traduit de l'allemand par Aline Weill, Paris, Payot \& Rivages, Paris, 2011, p. 45-46.

6. En référence à Angela Davis, Une lutte sans trêve, traduit de l'anglais par Frank Barat, Paris, La Fabrique, 2016. 
7. Daniel Bensaïd, op. cit, p. 252.

8. Ibid., p. 254.

9. Ibid., p. 250.

10. Propos de Kurt Tucholsky cités par Daniel Bensaïd, op. cit., p. 251.

11. Ibid., p. 254.

12. Giorgio Agamben, Qu'est-ce que le contemporain?, Paris, Payot et Rivages, 2008, p. 11.

13. Ernst Bloch, Rêve diurne, station debout et utopie concrète : Ernst Bloch en dialogue, Entretiens avec José Marchand (1974), traduits, présentés et annotés par Arno Münster, Paris, Editions Lignes, 2016, p. 126.

14. E. Bloch, Le Principe-Espérance, T.1., op. cit., p. 398.

15. E. Bloch, Experimentum mundi, Question, catégories de l'élaboration, Praxis, traduit de l'allemand par Gérard Raulet, Paris, Payot, 1981, p. 228.

16. Ibid.

17. Ibid., p. 304.

18. Ernst Bloch, Le Principe-Espérance, t. 3, op.cit., 305.

19. Ibid., p. 462.

20. Ibid., p. 326.

21. Giorgio Agamben, «De l'utilité et de l'inconvénient de vivre parmi les spectres » dans Nudités, traduit de l'italien par Martin Rueff, Paris, Payot \& Rivages, 2012, p. 58.

22. Ibid.

23. Ibid.

24. Ibid., p. 60 .

25. Ibid.

26. Ibid.

27. Ibid., p. 62.

28. Ibid..

29. Ibid., p. 63.

30. Ernst Bloch, Le Princpe-Espérance, T. 1, op.cit., p. 397.

31. Ibid, p. 398.

32. Ibid.

33. Ibid.

34. Jacques Derrida et Bernard Stiegler, Echographies de la télévision, Entretiens filmés, Paris, Galilée/INA, 1996, p. 135.

35. Ibid.

36. Tel aura été l'objet de l'article fondamental d'Adolfo Vera, «Le cinéma ou l'art de laisser revenir les fantômes : une approche à partir de J. Derrida ", in Appareil, $\mathrm{n}^{\circ}$ 14, 2014, mis en ligne le 12 décembre 2014, disponible sur : http://appareil.revues.org/2115.

37. Entretien de Jacques Derrida avec Antoine de Baecque et Thierry Jousse, « Jacques Derrida. Le cinéma et ses fantômes ", in Cahiers du cinéma, n 50, avril 2001, p. 78

38. Jacques Derrida, Spectres de Marx, Paris,Galilée, 1993, p. 21.

39. Ibid., p. 15.

40. Ibid., p. 15.

41. Ibid, p. 279.

42. Gregory E. Smoak, Ghost Dances and Identity, Prophetic Religion and American Indian Ethnogenesis in the Nineteenth Century, Berkeley-Los Angeles-Londres, University of California Press, 2006, p. 1.

43. Ibid., p. 2.

44. Ibid.

45. Ibid., p. 3.

46. Ibid., p. 165.

47. Ibid., p. 114. 
48. Ibid.

49. Ibid.

50. Ibid., p. 157.

51. Le film est visible sur le site de la Library of Congress : < https://www.loc.gov/item/00694139/>. 52. Dans ce film, la mort hante l'image à plusieurs niveaux. En plus de représenter une ronde funéraire quelques années à peine après la fusillade de Wounded Knee, la condition de vie des êtres au sein de la troupe de Buffalo Bill est à préciser. Comme l'écrit Sam A. Maddra : «Tandis que la Danse des esprits se propageait à travers les réserves des Lakotas, environ soixante-cinq d'entre eux, hommes et femmes, étaient en tournée sur le continent européen avec le Buffalo Bill's Wild West (...). Six interprètes indiens moururent en Europe, et les Américains lurent les descriptions des maladies et des soins inappropriés venant d'Indiens insatisfaits qui avaient quitté le spectacle, en plus des comptes-rendus d'Indiens autorisés à boire, parier et courir après les femmes » dans Hostiles? The Lakota Ghost Dance and Buffalo Bill's Wild West, Norman, University of Oklahoma Press, 2006, p. 63.

53. Ibid.

54. Ibid.

55. Kristin Ross, L'imaginaire de la Commune, traduit de l'anglais par Etienne Dobenesque, Paris, La Fabrique, 2015, p. 17.

56. Ibid.

57. Ibid., p. 43.

58. Daniel Bensaïd sé référant à René Scherer et Guy Hocquenghem dans Le pari mélancolique, op. cit., p. 256.

59. Kostas Axelos, Métamorphose, Clôture-Ouverture, Paris, Editions de Minuit, 1991, p. 133.

\section{RÉSUMÉS}

Face à la mélancolie comme dépréciation morbide, nous pouvons postuler avec Daniel Bensaïd l'existence d'une «mélancolie active » liée à la force critique du mélange des temporalités. Cette mélancolie singulière, "qui ne désarme pas ", est peut-être celle qui nous pousse à convier les spectres au cœur du monde contemporain, à réfléchir aux « images-posthumes » dont parle Ernst Bloch face à la condition larvaire que met en lumière Giorgio Agamben. L'hypothèse est de considérer le cinéma comme un espace potentiel où s'entretenir avec les esprits : c'est à partir de Ghost Dance de Ken McMullen (1983) au sein duquel apparaissent les fantômes de Jacques Derrida, de Pascale Ogier, des indiens Lakotas et de la Commune de Paris que nous tentons de l'expliciter.

\section{INDEX}

Index chronologique : XXe siècle, XXIe siècle

Mots-clés : cinéma, mélancolie active, image-posthume, spectre, larve, résistance amérindienne, Agamben Giorgio, Bensaïd Daniel, Bloch Ernst, Derrida Jacques, Ogier Pascale, la Commune, Ghost Dance, McMullen Ken

Index géographique : Europe, Etats Unis

Thèmes : cinéma et philosophie, politique contemporaine 


\section{AUTEUR}

\section{RAPHAËL SZÖLLOSY}

Raphaël Szöllösy est doctorant et enseignant en études cinématographiques à l'Université de Strasbourg. Son travail de thèse s'intitule Images en mouvement du monde morcelé : principe cinématographique pour faire face aux temps contemporains. Ses publications et communications ont exploré l'œuvre de plusieurs cinéastes issus de multiples contextes géographiques et sociaux du $20^{\text {ème }}$ et du $21^{\text {ème }}$ siècle (Pasolini, Amos Gitaï, Tariq Teguia, Jia Zhang-ke...) à partir des pensées d'auteurs inhérents à sa recherche centrale : depuis la philosophie d'Ernst Bloch jusqu'à celle de Cornelius Castoriadis et de Bernard Stiegler, comme la méthodologie d'Aby Warburg et de Georges Didi-Huberman, qui permettent de saisir le désarroi politique le plus actuel et de construire une réponse face à celui-ci. 